
internationales

vol. 30 - $n^{\circ} 2$ | 2014

Composer (avec) la frontière. Passages, parcours

migratoires et échanges sociaux

\title{
Quand les territoires circulatoires des transmigrants traversent des quartiers enclavés de villes moyennes françaises
}

When the "territoires circulatoires" of Transmigrants Come across Enclosed

Districts on French Middle Towns

Cuando los "territoires circulatoires» de los transmigrantes atraviesan

barrios aislados de ciudades francesas de mediana talla

\section{Tarrius Alain}

\section{OpenEdition}

\section{Journals}

Édition électronique

URL : https://journals.openedition.org/remi/6805

DOI : $10.4000 /$ remi.6805

ISSN : $1777-5418$

Éditeur

Université de Poitiers

Édition imprimée

Date de publication : 1 juin 2014

Pagination : 169-192

ISBN : 979-10-90426-22-1

ISSN : 0765-0752

Référence électronique

Tarrius Alain, «Quand les territoires circulatoires des transmigrants traversent des quartiers enclavés de villes moyennes françaises », Revue européenne des migrations internationales [En ligne], vol. 30 $n^{\circ} 2$ | 2014, mis en ligne le 01 juin 2017, consulté le 15 avril 2022. URL : http://journals.openedition.org/ remi/6805; DOI : https://doi.org/10.4000/remi.6805 


\title{
Quand les territoires circulatoires des transmigrants traversent des quartiers enclavés de villes moyennes françaises
}

\author{
Tarrius Alain ${ }^{1}$
}

L'observation de collectifs de transmigrants européens en provenance d'Afrique, du Proche et Moyen-Orient, des Balkans et du Caucase et, récemment (Tarrius, Missaoui et Qacha, 2013), de leur rencontre avec de jeunes habitants, descendants d'immigrés sédentarisés, enclavés dans des quartiers urbains français, est chose assez rare pour que je m'explique sur les préalables méthodologiques et théoriques mis en œuvre pour voir, décrire et analyser cet événement. En effet, les populations transmigrantes excèdent des frontières de chaque nation traversée ; elles n'ont aucun statut, juridiquement établi, sinon celui "d'étrangers de passage "; elles échappent donc au regard des instances publiques et leurs interactions avec des populations locales laissées pour compte de l'intégration n'occupent aucune place dans les curiosités et les intérêts politiques usuels. J'ai construit ces préalables en champ notionnel par d'incessantes négociations entre allers-retours de terrains et corpus sociologiques : la notion de paradigme de la mobilité, que je proposai en 1989, puis celle de territoire circulatoire, formellement construite en 1993, et souvent réexprimée depuis, en sont le centre. C'est grâce à elles que je visibilise, décris et analyse des faits sociaux originaux, relatifs aux migrations internationales et redevables de mouvements transfrontaliers constants : " paysages kaléidoscopiques ", selon l'expression de Merleau-Ponty $(1976)^{2}$ qui ne prennent sens qu'à partir de l'implication déterminée du chercheur.

1 Professeur de sociologie et d'anthropologie des migrations et de la ville, LISST (CNRS UMR 5193 - Université Toulouse II - Le Mirail) et MIGRINTER (CNRS UMR 7301 Université de Poitiers), Maison de la Recherche, 5 allée Antonio-Machado, 31058 Toulouse cedex 9 ; altarrius@gmail.com

2 Le philosophe décrit comment l'implication, de l'acteur-chercheur donne sens à des manifestations de la réalité apparemment disjointes ; problématique qui rappelle celle de Schütz (1988). 


\section{Construction d'un champ notionnel}

\section{Apparition d'une centralité économique urbaine, migratoire et internationale, masquée dans les années 1980}

C'est en 1984 que Gaston Deferre, maire de Marseille et ministre de I'Aménagement et de la Décentralisation, contacta mon laboratoire parisien ${ }^{3}$. II désirait comprendre pourquoi les résidents algériens d'un quartier central de Marseille, Belsunce, avaient inventé leur propre système de transports, à l'origine, affirmait-il, de rixes avec les taxis de la ville. J'avais récemment mené des recherches sur des microsystèmes de transports autoproduits ${ }^{4}$ (Barel, 1985), en marge de l'offre publique techno-produite. J'avais constaté combien les itinéraires, les horaires et les dimensionnements de ces services étaient adaptés aux rythmes sociaux (Gurvitch, 1963 ; Grossin, 1974 ; Mercure, 1979) caractéristiques des modes de vie des milieux désignés comme marginaux qui les produisaient. J'acceptai la proposition de l'édile marseillais, qui me permettrait, pensai-je, de compléter mes travaux par une observation en milieu urbain :

"La recherche doit être discrète. Des milliers d'Algériens arrivent à Marseille chaque semaine par air et par mer et se rendent dans ce quartier arabe du centre en empruntant des taxis clandestins : mes taxiteurs concurrencés par ces immigrés en viennent aux mains $n^{5}$.

L'opportunité de cette demande décida de mes orientations de recherche ultérieures, jusqu'à aujourd'hui. Je découvris, dans ce quartier désigné unanimement par les responsables politiques, administratifs et techniques de l'urbanisme marseillais, par les notaires et les agents immobiliers, comme celui de la déshérence, de l'impossible réhabilitation, le dispositif commercial le plus riche de la façade méditerranéenne française (Tarrius, 1987, 1995, 2007). Les rezde-chaussée des immeubles délabrés des XVII-XVIIIe siècles proches du VieuX Port, publiquement mis en vente, en 1987, autour de 1000 francs le mètre carré, se négociaient secrètement autour de 10000 francs entre Maghrébins, coût du foncier en vigueur dans les quartiers bourgeois de Marseille. 350 petites boutiques, exposant en vitrine des œillets de plastique et des couscoussiers d'aluminium, négociaient en réalité, et en arrière-boutique, de l'électroménager, de l'audiovisuel, des pièces de rechange de voitures, etc., pour un chiffre d'affaires global estimé à plus de 2,9 milliards de francs ${ }^{6} .700000$ Maghrébins $^{\circ}$

\footnotetext{
3 Département des études des mobilités urbaines de l'EPST INRETS.
}

4 Par des groupes aux contours communautaires affirmés : néo-ruraux de vallées pyrénéennes, Maghrébins en milieu périurbain.

5 Entretien téléphonique, mi-novembre 1984. La "reconquête " des quartiers arabes du centre-ville avait été le thème majeur d'une récente campagne électorale et le zèle électoraliste des taxiteurs agréés s'était révélé exemplaire, les désignant comme " clients " privilégiés du pouvoir municipal. "L'affaire est chaude ", insista le maire. Quelques mois auparavant, la municipalité avait fait enlever, quartier Bourse, près du site de mon étude, des barrières de planches décorées de paysages sahariens, chameaux et palmiers, entourant des travaux de fouilles archéologiques: de nouvelles palissades décorées d'igloos et de pingouins avaient été hâtivement érigées. Ambiance et contextes marseillais d'époque.

6 Par le bureau d'études SEDES de la Caisse des Dépôts et Consignations à la demande de la Chambre de commerce. 
passaient annuellement (1987) par ce dispositif commercial compensatoire des difficultés d'importation de l'État algérien : il s'agissait d'allers-retours par air ou par mer depuis l'Algérie et d'escales pour des émigrés en congés en France, en Belgique ou en Allemagne. Les approvisionnements étaient réalisés " en officiel » par des usines européennes écoulant des stocks d'invendus ou de séries à peine obsolètes, et en "souterrain " par des réseaux de passeurs de diverses nations ${ }^{7}$. À Belsunce encore étaient vendus, horsTVA, des produits alimentaires ${ }^{8}$ (Carreno, Hayot et Lesme, 1974) directement collectés chez des producteurs des régions voisines : fruits et légumes de Cavaillon à Nîmes, moutons de Sisteron à Apt. Les petits commerçants marseillais des divers arrondissements bénéficiaient de ces livraisons directes. Notre recherche décrivit une centralité commerciale internationale et locale en pleine expansion, au cœur de ce Marseille économiquement déclinant. Les multiples activités commerciales de cette centralité euromaghrébine n'étaient pas vues par l'officialité, comme amnésiées, alors même que les flux et les itinéraires des clientèles étaient clairement décrits. C'était la première identification européenne d'un " effet de mondialisation ", à l'initiative de populations en migration, conjuguant des échanges, du très proche au très lointain, qui échappaient aux initiatives d'acteurs politiques et économiques locaux et nationaux ; il s'agissait, dans I'histoire singulière de Marseille et de la France, d'un "retour de la colonie " (Braudel, 1979) impensable pour les populations locales et leurs édiles. II restait à rendre compte de l'évidente déconnexion entre la réalité des richesses, accumulées et cédées par de vastes et intenses mobilités, et l'invention collective de l'officialité, des désignations de déshérence du même quartier au cœur d'une métropole millionnaire appauvrie. Les données " objectives " de type statistique, INSEE par exemple, se révélaient particulièrement inadaptées. Ainsi, les variations du coût du mètre carré commercial maghrébin, précédemment signalées, n'existaient pas, laissant place à l'uniformité de la pauvreté supposée. Par exemple encore, les comptages de population faisaient état d'une masse de résidents immigrés pauvres et inactifs d'un quartier-dortoir en déshérence, là même où ils déployaient un intense dynamisme commercial international. Remplacer, affiner ou superposer des données statistiques ne permettait pas davantage de comprendre les valeurs et les usages différenciés des diverses populations présentes sur ce même emplacement (Gourdon, Perrin et Tarrius, 1995). Ma recherche devait donc permettre de comprendre les modalités du confinement urbain qui rendaient invraisemblables, pour un "Marseillais d'ici ", les initiatives de populations arabes pauvres, tout autant que les règles d'accessibilité d'un univers d'échanges souterrains superposé à la pauvreté urbaine ambiante ${ }^{9}$.

\footnotetext{
7 Par exemple, des réseaux sénégalais livraient des pièces de rechange de voitures contrefaites dans le Piémont italien, des Turcs des antennes paraboliques allemandes, des Marocains des tapis fabriqués en Belgique, et diverses marchandises espagnoles. L'électroménager venait d'usines françaises, allemandes et italiennes en achats groupés.

8 L'organisation des besoins de quotidienneté par et pour les 17000 résidents d'origine maghrébine qui peuplaient cet arrondissement de 27000 habitants, entre la gare SaintCharles et le Vieux Port, était déjà signalée en 1974.

9 La mairie de Marseille n'en demandait pas tant ; elle signifia brusquement la fin de la mission de 1984 par note aux agences d'urbanisme (1986) : " Monsieur Tarrius n'est mandaté par personne pour mener ses enquêtes ". Ce qui, évidemment, me valut des collaborations multiples.
} 


\section{Du paradigme de la mobilité aux territoires circulatoires: construction de deux notions nécessaires à l'analyse multi scalaire et multi située de la rencontre du global et du local}

\section{Espaces/temps sociaux, juxtapositions/superpositions socio-spatiales}

Les juxtapositions socio-spatiales sont un grand classique des approches de la ville : valeurs du foncier et regroupement des populations à partir de leurs conditions économiques, lieux des ségrégations, logiques foncières capitalistes, rôles dortoirs de quartiers versus rôles commerciaux et industriels, plutôt diurnes, mobilités spatiales intra ou périurbaines conçues comme strictement fonctionnelles, etc., nourrissent les dossiers d'aménagement d'intéressantes statistiques détaillées. Mais ces " mises à plat ", représentations de réalités urbaines à partir de préconceptions sur la claire lisibilité et paramétrisation de ce qui ferait "objectivement " ville, n'étaient pas à même de permettre la compréhension de mes observations. Et particulièrement celles qui décrivaient la superposition territoriale en œuvre dans ce quartier, redevables de vastes circulations de populations stigmatisées et de marchandises d'usages courants.

Le corpus des sciences sociales, ressource indispensable dès lors que la construction de notions originales s'impose pour rendre compte processuellement de réalités complexes ethnographiées, m'offrait deux intéressantes intuitions. Halbwachs, celui de La topographie légendaire des Évangiles en Terre Sainte (1941), bien différent du spécialiste durkheimien de la valeur foncière des années 1900-1930, identifiait en Palestine un phénomène de superposition : les implantations historiques longues, sur ce même espace des croyants des trois religions du Livre, avaient produit des désignations et des usages sociaux différenciés des mêmes lieux. Trois Palestine étaient en somme superposées, aboutissant à toute sorte de compromis entre les populations de croyants, aussi bien pour des usages spécifiques et communs que pour la désignation des lieux. Si la conception de la profondeur historique de ce phénomène et son actualisation dans la quotidienneté des échanges m'était apparue heuristique, par contre son enracinement, affirmé par Halbwachs, dans des mémoires collectives conçues comme des entassements mnésiques ne pouvait rendre compte des incessantes mobilités, locales, régionales, internationales, que j'observais sur mes terrains marseillais. La labilité de cette centralité enfouie, qui déjà produisait des rhizomes locaux, régionaux, internationaux, échappait à la compréhension proposée par Halbwachs. Les répétitions mémorielles ne rendaient pas davantage compte de l'originalité des productions de rapports sociaux ${ }^{10}$. II fallait dynamiser ces ensembles trop ethnographiques tant qu'on en restait aux descriptions de lieux, d'emplacements, d'itinéraires. Foucault, dans sa conférence inédite ${ }^{11}$ sur les hétérotopies, distinguait des constructions socio-spatiales singulières de leurs doubles temporels, les hétérochronies. Cette dissociation des mêmes territoires entre un univers fluide, organisé autour des temporalités sociales, des continuités processuelles et un univers figé par les contiguïtés

10 En outre, je voyais mal comment la mémoire collective selon Halbwachs expliquait les phénomènes d'amnésie observables dans ce quartier d'apparitions séculaires et phasées d'initiatives reconnues aux immigrés italiens, juifs et arméniens, et déniées aux immigrés maghrébins.

11 Prononcée en 1967 elle ne fut éditée qu'en 1988, après sa mort. 
spatiales, me parut particulièrement intéressante. Mais Foucault en resta à ses a priori sur la prévalence des formes et des ordonnancements spatiaux, et leur lisibilité immédiate, tels des paradigmes discursifs, sur les analyses processuelles, syntagmes sources d'imprévisibilités, craignait-il. II insista sur la durée moyenne, "à l'échelle d'évènements, d'étapes, d'une vie " des manifestations de ces dissociations. Là encore, les sens attribuables aux mobilités initiatrices $\mathrm{d}^{\prime}$ analyses multi situées et multi scalaires ${ }^{12}$, étaient occultés. Je retins toutefois la conjugaison des temps longs avec ceux de quotidienneté, proposée par Halbwachs, et la " méso durée " des phénomènes de dissociation entre espace et temps envisagée par Foucault, comme introductrices à une analyse processuelle des faits que j'observais : mobilités, interactions, échanges, éphémérité des situations exposées relevaient en effet des hiérarchies des temporalités plus que des ordonnancements spatiaux.

La phénoménologie, notamment la Krisis d'Husserl (1976), puis les conférences sur la technique d'Heidegger, et bien sûr les écrits de Merleau-Ponty et Jean-Paul Sartre sur les temps de I'engagement des acteurs sociaux - dont le chercheur -, m'avait initié aux lectures " déparamétrisées " et processuelles des proximités socio-spatiales ${ }^{13}$ faisant voisinage (Heidegger, 1958) aussi bien en situation de sédentarité que de mobilité.

Dès 1990 les réseaux de circulants, ouverts désormais, à Marseille, aux migrants de l'Est européen et à l'expansion migratoire marocaine ${ }^{14}$, produisaient des rhizomes du dispositif commercial de Belsunce dans les villes moyennes traversées par les divers entrepreneurs circulants. Ces nouvelles centralités commerciales multiples apparaissaient le long des itinéraires des nouveaux migrants, en Espagne sur le Levant, en France en bordure méditerranéenne puis dans le couloir rhodanien ${ }^{15}$, jusqu'en Allemagne et en Belgique.

\section{Le paradigme méthodologique de la mobilité}

Je mis en synergie ces contributions qui rendaient lisibles les superpositions socio-spatiales en termes de prééminence des continuités temporelles sur les contiguïtés spatiales. Je construisis le paradigme de la mobilité (Tarrius, 1989), comme univers notionnel hiérarchisé, à finalité méthodologique, afin d'observer et de décrire processuellement les productions sociales redevables des migrants, nomades-et-entrepreneurs, identifiés dans mon enquête marseillaise. Je proposai trois niveaux, trois échelles, des temps sociaux comme cadre méthodologique de description et d'analyse des faits de mobilités migratoires

12 Multi situées selon les parcours transnationaux et les frontières spécifiques aux transmigrants, multi scalaires, telles les échelles du global au local, du territoire circulatoire à l'étape, suggérant des superpositions socio-spatiales originales et complexes.

13 Les travaux de ces phénoménologues inspirèrent, dans les années 1970, ceux, intégrés dans les corpus sociologiques, de Jean-Claude Chamboredon et Madeleine Lemaire (distances sociales et proximités spatiales) et de Pierre Bourdieu (sur I'habitus, voir Husserl, 1931).

14 Entre 1991 et 1999, plus d'1 million de Marocains partent en migration verș l'Europe, alors que les Algériens, commencent à vivre un important repli : le " coup d'État légal " de 1989, contre le FIS allait entraîner de graves troubles.

15 Voir les travaux de Battegay (2003) sur le marché de la Place du Pont à Lyon. 
spatiales et sociales ${ }^{16}$.

Je préconisai dès lors pour l'approche de toute formation de migrants, en mobilité ou en étape, trois niveaux d'analyse:

- Une première identification des interactions de voisinage, par observation participante, démarche anthropologique nécessitée par les manifestations croisées d'altérités. Les temps sociaux de quotidienneté délimitent des territoires spécifiques et/ou communs, de sociabilités de voisinage ${ }^{17}$. Ce premier niveau de l'enquête permet de caractériser et choisir des situations d'interaction, particulièrement représentatives des productions sociales originales, situations d'échanges au sens goffmannien ${ }^{18}$ (Goffman, 1983 ; Winkin 1989).

- Puis après les identifications des micro-espaces de sociabilités, consécutives à cette première instance d'enquête, et le repérage d'individus témoins des rapports mobilité/sédentarité d'étape, j'aborde le deuxième niveau par des entretiens sur les trajectoires résidentielles ${ }^{19}$ : chaque localisation est conçue comme étape. Très rapidement se dégagent des finalités de ces mobilités résidentielles trop hâtivement décrites, dans les enquêtes statistiques, comme des effets de hasard et de contraintes foncières.

- Enfin, à partir de l'entretien, si possible de groupe, et de l'observation participante, j'identifie les possibles appartenances à des formes migratoires de type diasporiques créant légitimité dans le choix des parcours et des étapes au nom d'antériorités historiques familiales, régionales ou nationales. La consultation d'études historiques, de données d'état civil, de désignations toponymiques ${ }^{20}$, de témoignages d'érudits locaux est nécessaire.

Chaque fois que possible, je m'efforce de développer simultanément ces trois approches. L'exigence qui en résulte, pour le chercheur, est le mimétisme circulatoire et résidentiel des populations étudiées : habiter parmi elles, circuler avec elles ${ }^{21}$, la production de rapports sociaux originaux, exprimés dans des situations d'interaction, n'étant pas réservée aux relations de sédentarité. Les hiérarchies identitaires locales sont, elles aussi, réexprimées : "Ma famille est de Nîmes depuis avant l'arrivée des immigrés algériens ", propos courants pour dire sa préséance identitaire nîmoise, se voit opposer " C'est un des premiers

16 Étant bien entendu que toute traversée des espaces est indissociablement expérience des hiérarchies sociales et économiques, épreuve incessante du rapport entre identités sédentaires et altérités mobiles.

17 Les rythmes de fréquentation des zones commerciales, par exemple, permettent des regroupements identitaires, suivis de fréquentations dispersées, tout autant que les réunions associatives ou cultuelles, etc.

18 Pour la dimension sociologique de l'interactionnisme symbolique, et du sens sociologique des situations retenues, on lira Goffman (1983). La conjugaison des temporalités est fondatrice, chez ce chercheur, des situations d'interaction, manifestations de l'ordre social institué tout autant qu'en gestation (voir Winkin, 1989).

19 La consultation de fichiers de bailleurs ou d'EDF donne souvent accès à des suites d'adresses intéressantes pour choisir des personnes.

20 Par exemple, les noms donnés par d'anciens résidents à des sous-ensembles de quartiers, "carrés " de tombes, lesquelles ont l'avantage d'être datées et de préciser parfois le nom du lieu de naissance.

21 Nombreux sont les chercheurs sédentaires, travaillant sur les migrations à partir de données soi-disant objectives parce que statistiques qui ne peuvent voir et décrire les populations en transmigration, pas plus que I'INSEE ou Eurostat, fournisseurs de données, et qui ne savent comment les identifier et les " paramétriser ". 
Marocains à avoir fait étape à Alicante et à Perpignan avant de s'arrêter ici, à Nîmes, et de repartir vers Lyon et la Belgique " (Tarrius, Missaoui et Qacha, 2013) qui marque une autre préséance identitaire, dans la configuration du territoire circulatoire amalgamant les villes citées. J'ai formellement construit cette notion en 1993 (Tarrius, 1993) après l'avoir utilisée dès $1988^{22}$ (Tarrius, 1988) à partir de tel ou tel de ses attributs.

\section{Indissociabilité des échanges économiques et affectifs : intérêt d'un champ notionnel pour la lecture des continuités souterrain-officiel}

$\mathrm{J}$ 'ai pu élaborer la notion de territoire circulatoire à partir de deux ordres d'attributs : les premiers exposés ci-dessus comme constitutifs du paradigme méthodologique de la mobilité, les seconds tributaires de ma découverte des travaux de Park et notamment d'une notion intuitivement composée, obscure, mais heuristique : " moral area " ou " espace de mœurs ", selon une traduction d'usage fréquent, ou encore " district moral ", proposition d'Isaac Joseph ${ }^{23}$.

Une " moral area » ${ }^{24}$ (Hannerz, 1983 ; Park, 1955), ou " espace de mœurs " est une notion proposée par Park, un des fondateurs, fin des années 1910, de l'École de Chicago de sociologie et anthropologie urbaines ${ }^{25}$ (Thomas et Znaniecki, 1998). Elle désigne une conjonction fluide et imprévue des temps sociaux, des lieux, des mélanges de populations mobiles, généralement nocturne, susceptible de transformer les rapports sociaux tels qu'ils s'exposent lors des relations normées généralement diurnes. C'est le Chicago du début du XXe siècle qui posait la question : comment une accumulation-juxtaposition humaine, économique, culturelle, aussi hétéroclite que la capitale du Michigan faisait-elle ville? Comment ces populations d'immigrants italiens, polonais, allemands, irlandais et du Sud des États-Unis, produisaient-elles I'unité de la métropole alors même qu'elles se battaient en Europe ? Les comportements publics dérogatoires au " bon ordre diurne ", comme, à l'époque, la prostitution, les jeux d'argent, les consommations d'alcool en temps de prohibition, etc., provoquaient, la nuit tombée, grâce aux mobilités urbaines et périurbaines, aux brassages d'habitants aux profils contrastés, des proximités et des mélanges cosmopolites. Le fonctionnement diurne des institutions semblait bénéficier de ces effervescences, malgré leur nature apparemment " immorale ", déviante. L'observation empirique découvrait là une mise en œuvre de théories du fonctionnement social du philo-

\footnotetext{
22 Les travaux de Gildas Simon proposant une lecture dynamique des circulations migratoires dès 1987 et ceux d'Emmanuel Ma Mung sur les initiatives internationales déterminées de migrants internationaux $m^{\prime}$ encouragèrent dans cette voie socio-anthropologique complémentaire de leurs productions. Je rejoignis quelques années après, en 1994, le laboratoire Migrinter.

23 Professeur à I'université Lumière Lyon 2 de 1991 à 1993, j’ai co-animé le DEA de sociologie urbaine avec Yves Grafmeyer, Jean Métral, Alain Battegay et Isaac Joseph, traducteur-commentateur de I'œuvre de Goffman.

24 Hannerz, affirme que cette notion est la plus partagée par les sociologues de la ville qui se reconnaissent proches de l'École de Chicago.

25 Tripier présente, dans sa riche préface, ces deux chercheurs comme fondateurs de I'École de Chicago. C'est formellement vrai pour Thomas (1918, Université de Chicago) ; Znaniecki, disciple de Georg Simmel en Europe, marqua cette École, au-delà des années 1920, de l'influence de son maître.
} 
sophe du XIXe siècle, Friedrich $\mathrm{Hegel}^{26}$ et du sociologue Georg Simmel, qui I'un et l'autre ont tenté de penser l'unité du peuple allemand dispersé par I'histoire de la Mitteleuropa et, par endroits et par occasions, rassemblé sur le mode de fédérations migratoires inter-allemandes. Les approches usuelles " de la rationalité fonctionnelle ", disaient-ils, négligent une troisième dimension du changement social, souvent occultée par les débats politiques binaires, rapportés par la presse sur le ton de l'indignation sur des bases idéologiques et statistiques, à partir d'une dialectique simpliste du pour et du contre. Cette troisième dimension de la dialectique du changement, rassemblant " l'encore-enfoui ", le " non-admis " ${ }^{27}$ des comportements collectifs humains, serait déterminante des processus de changement longs et complexes ${ }^{28}$. Protégée, masquée par l'ordre officiel "de-ce-qui-peut-s'exposer ", elle en devenait d'autant plus redoutable. Pour le dire trivialement, dans le style des pionniers de l'École de Chicago, le partage nocturne du goulot d'une bouteille de whisky de contrebande dans les années 1920-1930 par le dirigeant d'entreprise et son boy, que la mobilité de I'un depuis sa villa et de l'autre depuis son taudis permettait sous l'égale injonction du même désir ${ }^{29}$, ce comportement-là était garant du bon ordre diurne. Le boy déployait alors l'ombrelle sur la tête du dirigeant à l'entrée d'un immeuble d'affaires, sans partage du tapis rouge, et la banque garnissait ses coffres-forts de leurs activités, diurnes comme nocturnes; de la même façon les escapades nocturnes vers les bars à femmes, contribuaient-elles à la richesse de certains autant qu'aux prêches des pasteurs, etc., éthiques sociales présentées comme antagoniques, opposées, selon la langue de bois publique, mais complémentaires et en continuité selon nos socio-anthropologues. Multiplions cela par les situations et les opportunités nées de ces moments nocturnes, sans oublier la circulation de l'argent en œuvre dans la grande métropole, et nous comprenons comment circule l'influence de populations infériorisées dans les rapports diurnes, tels les immigrants européens, Italiens et Allemands surtout, ou Noirs du sud des États-Unis. Bref, un monde fait de continuités dès lors qu'on le libère des barrières instituées par la "bonne morale " et ses images répulsives, ses frontières entre acceptable et condamnable, interdit, masqué. La description du Chicago de l'époque est évidemment exportable : la notion de " moral area " ou

26 Le " mouvement des jeunes socialistes ", se réclamant de Friedrich Hegel, avait gagné Chicago dès la fin du XIXe siècle.

27 Friedrich Hegel proposait l'usage commun de la langue allemande comme niveau profond de l'identité, et Georg Simmel identifiait des processus, souvent évolutionniste, de mutations identitaires (i.e. le passage de la communauté villageoise à la sérialisation de la métropole).

28 Le passage de la simpliste dialectique pour/contre/synthèse, qui résume souvent des lectures mécanistes du changement social, aux apparents pour/contre dissimulateurs des sens enfouis, non exposés, mais actifs, ouvre la voie d'une interprétation triadique du changement. Conjugaison complexe, mais inclusive des processus sous-jacents et en continuité avec ceux, visibles, qui les expriment tout en les dissimulant. La langue était donc pour Friedrich Hegel ce troisième élément, enfoui sous les identités nationales des dispersions allemandes, qui devait recréer le lien social, I'identité d'un peuple allemand. Pour Park les circulations des hommes, de leurs désirs, de leur argent, jouent ce rôle.

29 L'analyse marxiste inspirait également Park qui considérait la circulation de l'argent comme un aspect important de la moral area. Économies de l'argent et du désir étaient étroitement intriquées, de "l'encore-enfoui " au manifeste (du jeu d'argent, des trafics, à la banque, etc.) : continuités clivées artificiellement par le masque des discours de la morale bourgeoise. Cette intuition sera approfondie par l'École de Francfort, dans les années 1950. Marcuse (1963), en particulier, reformulera la théorie de la troisième dimension à partir d'une tentative d'unification des théories marxiste et psychanalytique. 
" espace de mœurs " est opératoire, à condition d'en revoir les éléments constitutifs au fur et à mesure du travail de l'histoire sur les contextes, ce qu'autorise la notion, au contraire du concept. La mondialisation, ses mobilités et ses réseaux, dessinait, dans mes approches, des configurations territoriales étendues en " moral areas " originales : les agrégations transfrontalières en particulier. Ces continuités entre activités souterraines et officielles, cette intrication insécable entre relations affectives et économiques, les mobilités à l'origine des mixités, des métissages parfois, et continuellement des cosmopolitismes, étaient bien celles que j'avais observées dans mes approches du comptoir maghrébin marseillais, en 1984-1985, puis dans les va-et-vient transnationaux marocains en $2000^{30}$, et enfin chez des Afghans et diverses populations balkaniques et caucasiennes, abordées après 2003 aux entours de la mer Noire.

\section{Des territoires de la mobilité}

La notion de territoire est aussi floue que celle d'identité ; elle exige, à chaque usage, un rappel de sa définition. A minima, nous dirons que le territoire est une construction consubstantielle de la venue à forme puis à visibilité sociale d'un groupe, d'une communauté ou de tout autre collectif dont les membres peuvent employer un " nous " identifiant. II est condition et expression du lien social. Le territoire est mémoire : il est le marquage spatial de la conscience historique d'être ensemble. Les États-nations ont proposé une organisation des territoires basée sur la présence de hiérarchies politiques dans la totalité de I'espace support aux sociabilités. Les réseaux que nous observons dérogent à cette construction politique et s'instituent ainsi en contre-modèle.

Pour nous donc, la mémoire en partage, qui permet d'affirmer une identité circulatoire, est extensive autant que le sont les territoires des circulations : elle n'est pas puits mnésique, elle signale non pas l'épaisseur des lieux connus, et les légitimités identitaires locales, mais les moments des négociations, des interactions, qui permettent de porter plus loin ses initiatives, de rencontrer, de traverser plus de différences, de multiplier les interactions en situation de mobilité. Cette mémoire collective est avant tout souvenir des accords de parole, des échanges d'honneur, des modalités transactionnelles qui fluidifient les circulations, qui permettent d'échapper aux régulations étatiques formelles, de contourner les règles de construction des frontières entre territoires nationaux et entre univers de normes. Les territoires circulatoires génèrent, en se déployant, leurs propres frontières fluides, abritant de très complexes univers cosmopolites : la notion de "réseau ", formalisatrice, est peu adaptée à la description de ces territoires, sinon comme descriptive de l'extérieur, des intentionnalités de divers acteurs circulants. L'association insécable des ressources affectives et économiques des territoires circulatoires constitue un milieu en expansion constante, relevant de la curiosité anthropologique, peu compatible avec la logique formalisatrice de la notion de réseau.

30 La notion de territoires circulatoires a inspiré, et inspire toujours, des travaux nombreux et intéressants ; les phénomènes de mobilisations transnationales qu'elle permet de décrire en incluant, au-delà des réseaux de l'économie souterraine, les singularités et l'épaisseur des rapports sociaux ouvrent la voie à une nécessaire anthropologie de la mobilité. 
Ces territoires, nous les nommons territoires circulatoires. Cette notion constate la socialisation d'espaces supports à des pratiques de mobilité. Elle introduit une double rupture dans les acceptions communes du territoire et de la circulation : d'abord elle nous suggère que l'ordre né des sédentarités n'est pas essentiel à la manifestation du territoire et des hiérarchies identitaires qu'il génère, ensuite elle exige une rupture avec les conceptions logistiques des circulations, des flux, pour investir de sens social le mouvement spatial. Le déplacement, qui ne peut dans cette perspective être considéré comme l'état inférieur de la sédentarité, confère, à ceux qui en font transitoirement leur principal lieu d'expression du lien social, le pouvoir du nomade sur le sédentaire : la connaissance des savoir-faire-chemin, condition de la concentration-diffusion des richesses matérielles et symboliques ${ }^{31}$, donne force sur l'ordre des sédentarités, et plus particulièrement sur sa manifestation première, l'espace urbain.

L'expansion de ces territoires, inséparable des solidarités qui les constituent en topiques d'échanges de haute densité et diversité, génère sans cesse de nouvelles connivences avec de nouveaux autres, fédérés au collectif circulatoire pour mieux transiter, atteindre des marchés, des emplois, des sites, de plus en plus lointains. II s'agit d'un monde des interactions créatives entre altérités. Les différences attachées à l'ethnicité en sont de plus en plus bannies dès lors que se manifeste cette éthique sociale intermédiaire. En somme, l'identité commune à tous les arpenteurs des territoires circulatoires est faite de la plus grande interaction possible entre altérités ; ainsi naissent continuellement des nouveaux territoires comme mondes cosmopolites mobiles.

La notion de territoire circulatoire habilite une démarche anthropologique étendue à la définition d'espaces relativement autonomes supportant des segmentations sociales, économiques et spatiales originales, générant ses propres frontières, spatiales et sociales, comme traverses de celles instituées par les nations. La mondialisation sur un mode ultra libéral, qu'inspirent les territoires circulatoires, contribue " par le bas " à la fin de l'ère des nations. La mobilité spatiale exprime donc plus qu'un mode commun d'usage des espaces. Les territoires circulatoires sont une topique intégratrice originale de populations souvent stigmatisées, " minorisées ", invisibilisées. Le cas marseillais précédemment relaté est emblématique de ces processus.

Une grande labilité caractérise les lieux d'articulation entre territoires circulatoires et espaces locaux, de telle sorte que tel emplacement, marché, rue commerçante, agrégé à l'espace des réseaux, peut en disparaître rapidement pour apparaître tout aussi rapidement dans un autre quartier de la ville, de la périphérie, de villes ou villages voisins, drainant les mêmes populations.

31 On comprend à quel point les TIC accompagnent et contribuent à l'expansion des territoires circulatoires. La lecture des productions de Diminescu (2005) est édifiante à cet égard. 


\title{
Quand les territoires circulatoires des transmigrants croisent les quartiers de relégation des immigrants
}

\author{
La mer Noire, I'ltalie du Sud, I'Andalousie, moral areas sur \\ les routes migratoires : des migrations ethniques \\ aux transmigrations cosmopolites
}

Les migrants internationaux mobilisés en groupes ethniques ${ }^{32}$ autour d'activités commerciales de vente, généralement parmi des populations pauvres ${ }^{33}$, de produits ou de services d'usages licites s'agrègent en groupes cosmopolites à l'occasion d'étapes de quatre à six semaines dans de vastes moral areas, zones de brassages intenses.

Nos recherches, sur les continuités migratoires méditerranéennes entre sud et est et nord, de 1985 à aujourd'hui, nous ont permis d'en reconnaître trois : la mer Noire, I'Italie du Sud, et I'Andalousie. Cela concerne les routes méditerranéennes : à partir de la mer Noire et de la Turquie, une autre grande route européenne existe, la voie du Nord, essentiellement structurée par des circulants turcs, qui agrègent des populations caucasiennes, balkaniques, esteuropéennes, vers I'Allemagne et les nations européennes nordiques. La mer Noire est la plus importante matrice des transmigrations vers l'Europe ; I'ltalie du Sud et l'Andalousie sont des moral areas complémentaires, intégrant aux transmigrants déjà en mouvement des nouveaux groupes régionaux, toujours sur le mode du cosmopolitisme de route. De ces trois étapes méditerranéennes des transmigrants convergent vers la région marseillaise, carrefour des territoires circulatoires.

\section{Territoires circulatoires tels que la Route des Sultans et la " route en pointillés ": quand les enclaves des sédentaires deviennent étapes des circulants}

Les nombreuses circulations portuaires sur la mer Noire préparent les transmigrants des nations pauvres de l'Est et du $S^{34}{ }^{34}$ à parcourir l'espace de libre circulation "Schengen ". Les transmigrants Afghans du poor to poor ${ }^{35}$ mondial de l'électronique, et d'autres migrants internationaux en " formations ethniques " qui la traversent une dizaine de fois, en un ou deux mois, jusqu'à

\footnotetext{
32 Dans nos recherches : populations ethniques relevant de désignations nationales, i.e. les Afghans, nationales transfrontalières, les Albanais, régionales, les Abkhazes, les Pomaks, régionales transfrontalières.

33 Clients pauvres pour les reventes illicites de produits électroniques (voir note infra, poor to poor). II en va autrement pour les activités illégales de prostitution et de revente des drogues.

34 II s'agit bien des transmigrants de la mobilisation commerciale internationale du poor to poor, et non des émigrants de la misère dont les itinéraires sont très différents (par exemple les Afghans en direction de Sangatte passent préférentiellement par la Grèce) ; pour cette problématique, voir les travaux de Smaïn Laacher.

35 Économie mondiale " pour les pauvres et par les pauvres " : mobilisation de migrants internationaux pauvres par les grandes firmes du Sud-Est Asiatique (SEA), via Dubaï : passeurs hors taxes et hors contingentements, de marchandises vers les nations pauvres et les populations pauvres des nations riches, voir Multitudes (2012) Transmigrants!, Multitudes, 49 .
} 
passer chacun environ 100000 dollars ${ }^{36}$ de matériel en Bulgarie, en ressortent en formations cosmopolites, Afghans-Géorgiens-Ukrainiens-Azéris-Kurdes, etc. En expérimentant les altérités multiples des relations dans les périmètres portuaires ils se dotent des attributs nécessaires à la transmigration vers I'Europe de l'Ouest : un pidgin universel, par " I'association de diverses expressions européennes sur fond de broken-english moyen-oriental " (un Kurde), des connaissances précises des logistiques de réapprovisionnement en route, et une identification des espaces de chalandise parmi les sociétés traversées. Dès Burgas ou Varna, ports bulgares, les Baloutches afghans et iraniens introduisent leurs nouveaux compagnons de route à la nouvelle entité cosmopolite des transmigrants européens. Ils agrègent à leur milieu de colporteurs transnationaux ${ }^{37}$ (Fontaine, 2008) une moitié de l'effectif des candidates balkaniques et caucasiennes à l'entrée dans l'eldorado prostitutionnel du Levant espagnol ; ils les rencontrent dans les ports de la mer Noire, ukrainien, tel Odessa, russe, Sotchi, géorgien, Poti, et turcs, Trabzon et Samson. Le temps de leur migration vers le Levant espagnol est identique à celui des transmigrants du poor to poor, avec qui elles sont souvent liées : elles participent pleinement aux interactions caractéristiques du territoire circulatoire. Pour l'autre moitié, elles sont encadrées par des réseaux criminels géorgiens qui gèrent leurs déplacements vers I'Italie du Sud, puis vers I'Espagne. Dans ce cas, psychotropes opiacés et femmes constituent la marchandise ${ }^{38}$ (Tarrius, 2013) de ces réseaux étroitement ethniques : nous sommes là en dehors de la problématique des territoires circulatoires. Par contre, les mobilités portuaires, dans les espaces consacrés à la foule des transmigrants primo arrivants en formations ethniques et à leurs divers échanges, à leurs multiples interactions, produisent rapidement des brassages, des mixités, des cosmopolitismes, des intrications entre nature affective et économique des relations, caractéristiques de la moral area décrite à Chicago par Park, les échelles spatio-temporelles des mobilités étant démultipliées dans le contexte de mondialisation ${ }^{39}$.

Tous découvrent que l'espace européen des Balkans offre des continuités territoriales musulmanes historiques, à travers la Bulgarie, la Macédoine, le Kosovo, le Monténégro et le Sud-Serbie jusqu'à I'Albanie, qu'ils dénomment la route des Sultans. Elle n'est pas parcourue selon les règles des logistiques usuelles d'économie de temps dans un espace contraint, mais selon des temporalités variables : traversées directes, sept jours, arrêts pour un travail saisonnier en compagnie des transmigrants du commerce en poor to poor, deux ou trois mois, arrêts peut-être définitifs pour une union entre un ou une transmigrante et un ou une résidente locale. Ces topiques, où les temporalités

36 Les estimations donnent 6 milliards de dollars d'électronique au total ainsi " passée " par la mer Noire vers l'Europe, 8 milliards de dollars par la voie Djedda, Dakar, Miami (Tarrius, Missaoui et Qacha, 2013).

37 Au service d'un capitalisme commercial ultralibéral (abolition des frontières et détaxations radicales), ces commerçants ressemblent beaucoup aux colporteurs préindustriels décrits par Fontaine.

38 Un gramme d'héroïne afghane de bonne qualité vaut 8 euros àTrabzon, 12 dans les Balkans et 40 en Europe de l'Ouest, procurant des bénéfices considérables que le travail sexuel des femmes, légalement déclarées en Espagne, permet de blanchir.

39 Voir Tarrius (2012 et 2013) où est décrite la mutation du groupe ethnique en milieu cosmopolite. 
sociales donnent sens aux parcours et aux étapes, modulent les espaces, observables par le paradigme méthodologique de la mobilité, c'est elles que nous nommons territoires circulatoires: les transmigrants y trouvent sur le mode de la mobilité le lieu-mouvement de leur intégration de commerciaux du système mondialisé du poor to poor. L'expérience du cosmopolitisme que permettent ces parcours, avant d'arriver au deuxième balcon d'Europe ${ }^{40}$, sur les côtes adriatiques d'Albanie, est définitivement celle de la transmigration. La mise à distance des institutions étatiques, et la proximité des populations grâce à une production originale de rapports sociaux dans l'interface mobilité/sédentarité, en est la caractéristique que nous détaillerons dans la dernière partie de l'article. Les jeunes femmes, mobilisées pour le travail du sexe, partagent ces expériences de circulation et en demeurent liées à l'univers des transmigrants commerciaux : désormais, pour celles-ci, tout espace sédentaire devient étape, passage, et dès l'arrivée dans une destination convenue, la sortie est négociée, des Balkans vers I'Italie, puis le Levant espagnol, puis les nations permissives d'Europe du Nord et enfin, le retour. Des médecins proche-orientaux, surtout syro-libanais, après un stage à Sofia ${ }^{41}$, se joignent aux commerçants à destination de villes italiennes, françaises et espagnoles, dans l'espoir d'une embauche dans un dispositif médical ${ }^{42}$. Ces médecins ne partagent pas les parcours initiatiques des ports de la mer Noire : ils rejoignent la transmigration, sur la route des Sultans à partir de Sofia, grâce à leur grande proximité des Syriens commerçants, clients des transmigrants du poor to poor. C'est ainsi que le milieu des transmigrants agrège, à partir des Balkans et grâce au savoir relationnel acquis dans les ports de la mer Noire, divers groupes pour les tournées européennes.

La route des Sultans, se transforme, une fois passée l'Adriatique, et à partir de brefs séjours à Bari, Brindisi ou Tarente, en "route en pointillés ". Très rapidement, entre 2003 et 2007, les transmigrants ont repéré, dans les villes des nations ouest-européennes, les quartiers enclavés, peuplés surtout de leurs clientèles pauvres, qui se confondent en grande partie avec des Maghrébins sédentarisés après la grande immigration pour la force de travail sédentaire. Trans- et immigrants se croisent, fraternisent et inaugurent une nouvelle histoire migratoire.

\section{Dispositifs marocains locaux comme étapes sur les territoires circulatoires de la " voie méditerranéenne " et effectifs des transmigrants dans le carrefour méridional français}

En France les singularités de la transmigration maghrébine, marocaine surtout, qui, à partir de 1990 (voir supra), a créé les territoires des circulations

40 Le " premier balcon d'Europe " est situé, pour les migrants proches, moyen-orientaux et caucasiens, le long des côtes turques, géorgiennes et russes de la mer Noire.

41 Les accords en vue de perfectionnements de formation, passés entre "pays frères ", dans ce cas la Bulgarie et la Syrie, dans les années 1970, avaient institué ces échanges. Fin 1990 après l'effondrement du régime socialiste, 3000 d'entre eux, souvent parents de bazaris de Damas, restèrent en Bulgarie pour ouvrir des commerces d'électronique, de bijoux, ou encore des pharmacies. Les accords de coopération médicale ont été maintenus : étudiants et commerçants entretiennent un courant migratoire important parmi les classes moyennes syriennes.

42 Les diplômes des médecins maghrébins, libanais, syriens, permettent à leurs titulaires une embauche assortie d'un protocole de validation de leur formation. Généralement cette transition dure trois années d'un dur travail avec un statut hospitalier infériorisé. 
commerciales ouest-européennes sud-nord ${ }^{43}$ facilitent ces rencontres : les appartements occupés, pendant les années 1970-1980, lors de la migration industrielle et, dans le Midi, agricole, ont souvent été conservés lors du passage aux activités transmigratoires, avec les droits liés à la sédentarisation longue ${ }^{44}$. Les territoires circulatoires marocains, institués dans les années 1990, sont devenus ceux parcourus ultérieurement par les migrants passés par la mer Noire : pourtour européen du Bassin ouest-méditerranéen et couloir rhodanien avec I'extension jusqu'en Belgique et en Allemagne (Tarrius, 2007). En effet les années 1990 ont vu l'entrée en migration européenne de plus d'1 million de Marocains ${ }^{45}$, dont une grande partie sur le mode du transnationalisme commercial ; en même temps plusieurs dizaines de milliers d'immigrés marocains, résidents anciens, transfèrent vers les mêmes activités nomades.

Des appartements, une quarantaine de F4 et F5, dans des quartiers de HLM ou dans des enclaves centre-urbaines pauvres, utilisés par les migrants marocains sédentarisés, sont devenus, au fur et à mesure de leur passage des activités sédentaires aux activités commerciales transnationales, des dépôts de marchandises, mais aussi des lieux d'accueils de tous les circulants transmigrants.

Deux enquêtes, en 2009 et en 2012, nous ont permis d'identifier les villes moyennes qui, en grande région marseillaise, abritent ces appartements d'accueil des divers transmigrants (Tarrius et Bernet, 2010 ; Tarrius, Missaoui et Qacha, 2013). II s'agit surtout de Nîmes, Arles, Beaucaire, Avignon, Carpentras et Cavaillon. Plus de quarante appartements, au total, sont devenus lieux d'accueil de ce carrefour qui non seulement brasse transmigrants de l'Est et du Sud, avant leur montée vers l'Europe du Nord, mais encore permet une osmose entre circulants internationaux et résidents locaux, entre transmigrants et immigrés, et leurs descendants sédentaires.

Le carrefour des territoires circulatoires en provenance de l'Est, transmigrants du poor to poor par la mer Noire, médecins proche-orientaux, et ceux remontant du Sud-ouest, Marocains et jeunes femmes des Balkans et du Caucase quittant les clubs prostitutionnels espagnols pour les nations permissives d'Europe du Nord, est situé dans la " grande région marseillaise ". Dans des villes moyennes hors de la métropole.

Les effectifs des transmigrants concernés selon nos enquêtes :

- Les Marocains : de 3000 en 1991, à 30000 en 1993 et 120000 en 1995, le " point de stabilité " s'est établi, au début des années 2000 autour de 130000 transmigrants par an effectuant au moins deux voyages mensuels à travers trois nations européennes (Tarrius, 2002). II s'agit de l'effectif le plus nombreux et le plus ancien. Depuis six années, ils se " chargent ", auprès de clubs pros-

43 Les implantations commerciales " autoproduites " surtout algériennes tournées vers le commerce transméditerranéen, telles Belsunce à Marseille ou la Place du Pont à Lyon (Battegay, 2003), sont largement transférées, à partir de 1990, aux Marocains. Le " coup d'État légal " contre le FIS, en 1989 en Algérie, plaçant les premiers commerçants, Algériens, en position d'infériorité. On observe par la suite peu d'initiatives transmigratoires commerciales algériennes en Europe.

44 Notamment, bien sûr, celui de circuler dans I'espace Schengen.

45 Source : Centre Mohamed V pour les migrations. 
titutionnels, de marchandises passées par Dubaï, aussi bien lors de la montée vers la Belgique que du retour vers le Maroc. Les relations, précédemment signalées, nouées entre les transmigrants du poor to poor et les jeunes femmes, le long des territoires circulatoires des Balkans, aboutissent à cette extension des réseaux du poor to poor mondial. Dans l'étape des villes méridionales françaises ils livrent des produits précédemment acquis en Espagne, ou, lors des redescentes, en achètent d'autres aux transmigrants pratiquant une halte dans les appartements. Ces haltes concernent environ 18000 d'entre eux.

- Les Afghans et leurs associés : lors de nos premières identifications de ces transmigrants, en 2001, nous en comptions 300 au passage de la frontière franco-espagnole du Perthus, et en 2005 plus de 25 000. Lors de nos enquêtes de 2012 en grande région marseillaise, nous avons estimé autour de 8000 leurs passages annuels dans les appartements du carrefour méridional français. Ces lieux d'étape, dotés de dispositifs de communications électroniques, leur permettent de planifier leurs déplacements vers la route du Nord, comme nous le verrons plus avant.

- Les médecins proche-orientaux, dénommés "docteurs égyptiens ", que nous avions identifiés dans nos enquêtes de 2008 à Sofia au nombre de quelques centaines se dirigeant vers I'Italie, la France et l'Espagne, étaient environ 6000 lors de nos comptages de 2012. 4000 circulaient entre les trois nations en tournées annuelles d'environ huit mois, et 2000 tentaient d'entrer dans les dispositifs médicaux. Environ 300 circulaient dans le périmètre des régions PACA et Languedoc-Roussillon et une centaine, soit deux par mois, fréquentaient les quarante appartements du "périmètre marseillais ", animant les " dispositifs médicaux " que nous allons détailler. Sur ce modèle, plusieurs centaines de dentistes syriens, exerçant en Bulgarie et Roumanie, circulent, le long des étapes de Roms, des Balkans, au nord de I'Italie et en France méridionale, jusqu'en Guyenne ${ }^{46}$.

- Les femmes, au nombre d'environ 3 000, originaires des Balkans se dirigent vers l'Europe du Nord après un séjour de trois à cinq années dans les clubs prostitutionnels du Levant espagnol. Elles sont souvent accompagnées de parentèles des villes ou villages d'origine et parcourent les routes et autoroutes françaises durant six à neuf mois (Tarrius et Bernet, 2014). Elles trouvent auprès des appartements d'accueil des transmigrants, qu'elles ne fréquentent jamais, une assistance logistique pour l'organisation de leur travail. Elles habitent passagèrement les villes et villages proches de leurs lieux de travail.

\section{Déploiement de dispositifs populaires médicaux à la croisée des circulations et des sédentarités migratoires : résilience populaire en temps de crise}

\section{Étapes et usages des TIC}

À l'arrivée en région marseillaise les transmigrants proche et moyen-orientaux, caucasiens et balkaniques, fédérés durant leurs traversées de la mer Noire, ont parcouru, par la "route des Sultans ", la Bulgarie, la Macédoine et

46 Signalés par Alexandra Clavé-Mercier, doctorante en anthropologie sociale à I'université de Bordeaux Segalen. Thèse en cours : La " question Rom " en Aquitaine : politiques d'accueil et dynamiques identitaires. 
l'Albanie ${ }^{47}$, puis, par la " route en pointillés ", des villes italiennes, dont Naples et Gênes, et françaises, Nice, Toulon et Marseille. Le " mano a mano " et le " tombé du camion " ont épuisé leurs réserves de matériels électroniques passés en free tax par Dubaï et reconstituées à Durrës. Dans les appartements dédiés à leur accueil dans ce premier grand carrefour des transmigrations, ils rencontrent les leurs passés, de pointillé en pointillé, par les territoires circulatoires de l'Europe du Nord, la " voie turque " dit-on, qui s'apprêtent à poursuivre la route soit vers I'Espagne, soit vers la mer Noire, par la route méditerranéenne. Ils apprennent de vive voix où précisément effectuer leurs prochaines transactions et les ressources relationnelles du territoire circulatoire à venir. Ils rencontrent aussi les femmes qui, après des séjours de trois à cinq ans dans les clubs prostitutionnels du Levant espagnol, rejoignent, souvent en compagnie de parentèles des lieux d'origine, les nations permissives nord-européennes avant de retourner chez elles dans le Caucase et les Balkans. Elles effectuent la transmigration française sur les mêmes territoires que les transmigrants du poor to poor, en six ou neuf mois, travaillant le long des routes et des aires d'autoroutes, souvent accompagnées par des Géorgiens, employés des réseaux criminels prostitution et drogues - russo-italiens ${ }^{48}$, qui les " protègent " le long de leurs déplacements. Elles se tiennent à distance des appartements d'accueil, mais en reçoivent, nous le verrons plus avant, une aide logistique appréciable. En contrepartie elles permettent l'acheminement du dernier réapprovisionnement en marchandises passed by Dubaï arrivées sur le Levant à Alicante, Tarragone ou Barcelone. Elles sollicitent, au village frontalier de la Junquere, centralité européenne de la prostitution - 462 femmes - et des camions -3600 arrêts par jour -, des camionneurs pour livrer les quarante-deux appartements du triangle Avignon, Arles, Nîmes. Les Marocains commerçants transmigrants, généralement propriétaires ou locataires officiels de ces appartements situés au cœur de leur territoire circulatoire, du Maroc à la Belgique et l'Allemagne, enrichissent ce cosmopolitisme migratoire et donnent des conseils pour les rencontres commerciales nord-européennes avec des Turcs. Enfin les médecins sud-méditerranéens donnent là leurs consultations.

Les " appartements-étapes " recensés dans la grande région marseillaise gèrent cette vaste dynamique grâce à environ 200 jeunes, filles et garçons, des mêmes quartiers. La moitié de cet effectif se consacre aux communications électroniques (TIC), Internet et Skype ${ }^{\circledR}$, qui permettent de maintenir des liens constants entre les passagers des territoires circulatoires, leurs vis-à-vis sédentaires, leurs familles, etc. unissant la vaste fédération transcontinentale des territoires circulatoires. L'autre moitié effectue diverses tâches de proximité, notamment dans le domaine de la santé, les rendez-vous avec les médecins itinérants, les commandes de médicaments sur Internet, les suivis familiaux. Les femmes, en transit du Levant à l'Europe du Nord, ont recours à ces services TIC pour gérer leurs rendez-vous routiers. Les revenus fournis par ces nombreux

47 À leur arrivée au port de Durrës, en Albanie, ils se réapprovisionnent, autant que nécessaire, en produits " made in SEA ", arrivés du Golfe en cargos. Ils les revendront dans les quartiers urbains enclavés de la "route en pointillés ", en Italie, " mano a mano ", puis en France à Nice, Toulon et Marseille, "tombés du camion ".

48 Le terme milieu criminel russe désigne, pour ces populations, une fédération de groupes criminels de Géorgie, d'Ukraine et de la zone russe riveraine de la mer Noire (ports de Poti, Odessa et Sotchi). 
usagers, pour les services rendus, servent à rétribuer les jeunes employé/e/s. En somme, il s'agit d'une entreprise spécifique à ce carrefour des territoires circulatoires, des migrants-colporteurs en mobilité, recrutant son personnel parmi les descendants de migrants sédentarisés. Des quartiers en déshérence de ces enclaves urbaines sont, en quelque sorte annexés aux territoires circulatoires. Chaque jeune demeure de six à neuf mois dans ces fonctions avant de rejoindre les transmigrants sur les voies européennes. Il dispose alors d'une connaissance précise des situations transactionnelles qu'il devra aborder, et d'une liberté de circulation dans l'espace Schengen plus importante que celle de ses hôtes des territoires circulatoires.

\section{Un cas de résilience populaire en temps de crise : l'internet médical et les dispositifs de santé autoproduits dans le carrefour migratoire méditerranéen}

Trois situations et extraits d'entretiens permettront de saisir les contours d'une telle initiative, à partir des expressions-mêmes utilisées par les jeunes acteurs impliqués.

- Aïcha et Fatiha ${ }^{49}$ ou le paradoxe des " docteurs égyptiens " : archaïsme du diagnostic, modernité des médications:

"[Aïcha :] La nécessité d'organiser les passages des "docteurs égyptiens" est apparue il y a trois ans, à Arles, à Nîmes et Avignon. Les premiers [médecins] qui sont arrivés, surtout Syriens et Libanais, accompagnaient des Irakiens kurdes, des Afghans, ou des Turcs qui passaient par l'Albanie et I'Italie, plutôt des Arabes de la région d'Adana. C'étaient des vrais médecins formés à Damas, à Beyrouth et pour certains à Sofia, en Bulgarie. Ils essayaient de trouver un travail dans les hôpitaux : ils savaient que s'ils étaient embauchés c'était pour un travail déqualifié, mais ils espéraient passer des concours et, en quelques années, devenir de vrais docteurs des hôpitaux. Mais ce n'était pas aussi simple : travail de nuit, dans un milieu hostile ; ils représentaient la dégringolade des statuts de soignants français, comme cela avait été le cas chez les ouvriers trente ans avant, ils parlaient peu la langue, et étaient surchargés de gardes de nuit, etc. [...] Alors certains ont demandé aux associations proches des mosquées si elles désiraient des "soins musulmans" et surtout des visites sans dénudation des femmes. Comme ils travaillaient à l'hôpital de Marseille, tous les responsables religieux ont eu confiance ; mais ils exerçaient dans les villes en dehors de Marseille, par prudence. C'est

à Nîmes et Arles qu'ils ont commencé. [...] Les habitants, qui venaient en consultations familiales, dix personnes au maximum pour une heure disaient "les docteurs égyptiens", ce qui inspirait le respect. Après les croyants c'est tout le monde qui est venu dans le quartier; tu n'avais pas à te déplacer pour aller de plus en plus loin, et s'il fallait aller à I'hôpital ou chez un spécialiste ils te le disaient honnêtement. [...] Et puis, ils ont été rapidement appelés partout; alors ils ont rejoint les appartements qui servent aux Marocains du commerce en fourgons [les transmigrants] à Nîmes, Arles, Beaucaire et Avignon. [...] Alors, il a été clair que ce triangle de villes était le centre, la plaque tournante de tous ceux qui prennent la route, docteurs, commerçants ou femmes; au début on comptait chaque année le passage d'une centaine de docteurs ; aujourd'hui, avec ceux qui vont en Espagne et ceux qui remontent vers l'Allemagne, ou en descendent, c'est plus de

49 Aïcha et Fatiha sont deux jeunes " assistantes médicales " attachées à un dispositif autoproduit, avec I'aide d'une association cultuelle musulmane locale, autour des médecins transmigrants proche-orientaux, consultant dans trois quartiers d'une ville en région PACA. 
1000 de docteurs qu'on croise, presqu'un par semaine par appartement; pour la région de Toulon à Montélimar, Montpellier et Béziers, il faut compter environ 1000 passages par an avec ou sans arrêt pour consulter, de deux jours à une semaine. [...] Mélangés avec ceux du commerce seulement à Nîmes, Beaucaire, Arles et Avignon, puis dans les locaux des associations, ou des mosquées, ou chez des particuliers, qui gagnent un peu d'argent. En tout je compte vingt-sept lieux pour les consultations collectives; Fatiha, elle, qui livre des médicaments dit qu'il y en a plus de quarante dans la même zone. [...] Quand

ils s'arrêtent dans les appartements ou les maisons des Marocains, c'est souvent qu'ils $s^{\prime}$ entendent avec des commerçants pour faire la route avec eux.

[Fatiha :] Quand on en parle comme ça, surtout des diagnostics avec les pouls, pour ne pas dénuder les femmes, on pense à des guérisseurs ou à des charlatans. Pas du tout, ils ont des formations réputées [...] et tous disent combien il est difficile d'entrer

en médecine à Damas ; il faut être les meilleures mentions au bac. [...] Alors, ce qui frappe le plus, c'est de les voir comme des guérisseurs et de constater qu'ils utilisent les médicaments les plus récents, qui ne se vendent pas encore en France. Ça fait partie de notre travail de chercher sur Internet les médicaments qu'ils demandent. Ils lisent tous, en anglais, les revues spécialisées américaines et canadiennes et ils connaissent

les médicaments les plus récents, qu'on n'aura en France que dans quatre ou cinq ans. [...] Ils nous ont appris aussi à communiquer par Internet avec des docteurs des grands labos, pour la compatibilité entre médicaments, pour les variantes d'une molécule mieux adaptée à tel ou tel cas, et quand ils sont partis nous questionnons chaque fois qu'il peut y avoir un effet indésirable. [...] Alors, vous comprenez qu'il y a des filles au local, pour organiser les passages, d'autres aux commandes de médicaments et autres questions par informatique, d'autres aux distributions et au suivi à domicile des clients. Dans le triangle d'Arles, Nîmes et Avignon, dans la vingtaine d'appartements concernés, on compte plus de quarante filles aux ordis, autant aux distributions de médicaments et aux suivis et une vingtaine, une par appartement, à l'organisation, comme Aïcha. [...] On commande par Internet et [on reçoit] par la poste les médicaments américains les plus récents, qui ne seront vendus en France que dans des années.

[Aïcha :] On est débordées parce qu'il y a de plus en plus de clients qui ne voient pas les docteurs et qui veulent les mêmes médicaments que des voisins, parce que, aussi, il faut aller en chercher à Gênes ou à Barcelone où ils arrivent en quantité. [...] Alors, voilà ce qu'on a décidé : certaines parmi nous s'installent en solo et se consacrent aux commandes et redistributions de médicaments. Nous les assurons de notre clientèle, mais nous leur envoyons tous ceux qui veulent les médicaments sans passer par les docteurs. [...] C'est à Nîmes, dans les environs, qu'une de ces filles installée comme commerciale, a commencé à livrer des toubibs français à la recherche de produits américains récents. [...] Si ça continue, c'est les demeurés qui iront chez le pharmacien. D'autant plus qu'aux ordis, ils cherchent des génériques et en trouvent à quart de prix. Les copines travaillent de six à huit heures par jour, y compris le samedi, et parfois le dimanche pour les responsables de l'organisation ; c'est bien payé, directement par les docteurs [...] ; celui qui ne paie pas ne revient pas ici, ni dans la zone. Parmi nous certaines travaillent moins et profitent de leur expérience pour faire des études genre infirmières ou commerciales médicales. [...] Mais ce n'est pas comme les garçons, nous ne prenons pas la route ". 
- Azzedine, entrepreneur d'un appartement-étape du carrefour migratoire :

"À Nîmes, je dors dans notre appartement ${ }^{50}$, je me lève vers 6 h, moment de départ des fourgons, et je me couche aux dernières arrivées vers $11 \mathrm{~h}$ du soir ; le temps de manger tous ensemble, et au lit.

Tu veux savoir ce que je fais? Toujours plus, toujours en désordre; [...] bon il y a les dedans et les dehors. Dedans, deux femmes viennent le matin, pour la vaisselle de la veille, la propreté, le linge et la préparation des repas : un chaud à midi, un froid le soir.

Et puis c'est difficile, tout change selon les arrivées et les livraisons. II faut dire qu'on ne fait pas les rendez-vous des femmes de la route, c'est Beaucaire, Arles ou Avignon qui s'en occupent. Par ici et tout autour d'Arles, les maquereaux ukrainiens et géorgiens sont trop mal vus. [...] Par contre les docteurs et les médicaments c'est surtout nous et Avignon. Alors là, tu connais puisque tu as parlé à Aïcha et Fatiha ; ce que nous avons en plus c'est beaucoup de clients qui sont pas Arabes, qui ne passent pas par les docteurs

égyptiens ; ce sont des clients docteurs français qui nous passent des commandes délicates aux USA et au Canada. [...] En clair les toubibs d'ici, des HLM, s'en vont à cause de nous, les gens font plus confiance aux docteurs égyptiens qu'aux charlatans des cités en blouse blanche; et les toubibs qui sont pas d'ici, mais des beaux quartiers, spécialistes et compagnie, sont nos clients. Ils veulent tel nouveau médicament qui n'a

pas encore de générique. C'est très difficile parce qu'il faut être sûr des labos, mais attention : pour eux on ne cherche pas les meilleurs prix, brésiliens ou indiens. [...] Alors la fille qui cherche ouvre Merx-Canada : il y en a un paquet, des faux et le vrai; alors, va savoir ? Une seule méthode : tchatcher avec les docteurs du site ; les faux n'en ont pas ; repérer bien les liens : si tu vois apparaître .php ou .ru ou... tu te tires, c'est des voyous.

Nous avons six filles et toute une pièce pour le médical. [...] Moi, ce qui m'intéresse, $c^{\prime}$ est les marchandises et, certaines nuits, les rendez-vous des femmes sur l'A9-elles

travaillent dans les cabines des camions sur les aires de l'A9 - et sur les routes qui descendent vers Arles et Fos ou qui sont parallèles à l'A9 [...]. On est trois garçons aux manettes. Mais les marchandises c'est le plus important. Il y a tellement de métiers et $d^{\prime}$ origines qui se croisent, et qui se connaissent, que tu te croirais en plein boulevard du centre-ville, avec une population qui coule comme un fleuve".

- Samia signifie une modalité de repli du dispositif médical officiel :

Une jeune fille, qui travaille pour le dispositif médical d'un de ces appartements d'accueil, a décelé une " erreur " de prescription du médecin généraliste libéral de son quartier lors du traitement de la goutte d'un de ses voisins : celui-ci venait d'être opéré d'un cancer de la prostate et la prise du médicament pour la goutte semblait provoquer de graves troubles digestifs. L'adolescente, en lisant les mises en garde sur Internet découvrit qu'il était fortement décommandé d'administrer ce médicament (colchicine + poudre d'opium) pour la goutte en cas de "pathologies de la prostate ". La famille en fit part au médecin, qui répondit que de toute façon la prostate n'avait rien à voir avec la digestion. Catastrophe, la mise en garde Internet signalait à " effets indésirables " des

50 Azzedine a vingt-six ans. Cadet d'une fratrie de sept enfants, il vit entre Nîmes et Fès, ville où la famille s'est réinstallée en 2008 ; le père, Mohamed, a pris sa retraite cette année-là et se consacre depuis à plein temps à la transmigration commerciale, par l'Espagne, la France et la Belgique. II a gardé la location de l'appartement familial à Nîmes: "parce que c'est au centre de tous les passages, comme Avignon ". Azzedine et son frère Mourad, trente ans, assurent la gestion de l'appartement nîmois désormais devenu lieu d'accueil. 
" troubles graves de la digestion " : un " médecin référent ", de la firme qui produisait ce médicament, consulté en urgence par la jeune fille sur un site pharmaceutique Internet canadien confirma cette mise en garde. Le médecin local fut totalement discrédité auprès de très nombreuses familles. Il interpela la jeune fille à l'origine des informations, au pied de son immeuble et lui demanda de ne pas répandre des rumeurs : celle-ci, devant un public qui se densifiait de minute en minute tint tête :

"Tu devais donner de la colchicine simple et non du "colchimax®". À moins que tu sois un dealer d'opium. [...] Et si tu avais eu cette personne dans un examen quand tu faisais tes études, tu aurais été collé ".

Les applaudissements d'une foule d'une trentaine de personnes empêchèrent le praticien de répondre. Des descriptions de scènes identiques me furent rapportées par des témoins de l'altercation. Le médecin lui-même me confia que les conséquences de ce conflit étaient graves pour lui :

"Elle a vidé mon cabinet et rempli sa cuisine. [...] De toute façon je ne resterai pas ici ; les adolescentes avec Internet, les "médecins égyptiens" plus ou moins formés au Maroc, qui maintenant passent toutes les semaines pour des consultations collectives, les vieux qui reviennent du bled avec des herbes. C'est la vie tribale et les chamans qui

se multiplient $"$.

Ainsi se confirmait une spirale de déclin des dispositifs officiels et de succès des dispositifs initiés le long des territoires circulatoires comme résiliences populaires en temps de crise.

\section{Conclusion : généralisation des poor to poor et nouvelle figure de l'étranger ?}

De grands laboratoires de l'industrie pharmaceutique mondiale suivent, semble-t-il, les stratégies commerciales des majors de l'électronique du Sud-Est Asiatique $^{51}$ : implantation de l'économie mondiale " horizontale " du "poor to poor " ou " pour les pauvres par les pauvres ". À partir de Dubaï et Koweit City, qui, en toute légalité OMC, importent des produits électroniques du SEA (Sud-Est Asiatique) à prix de fabrication, totalement détaxés, les transmigrants ${ }^{52}$ pauvres, par la voie de Djedda pour l'Afrique, et de la mer Noire pour l'Europe, charrient ces marchandises. Qui serait plus qualifié qu'eux pour offrir aux firmes mondiales le vaste marché des pauvres, leur milieu naturellement proche, en passant, à leurs risques, des marchandises totalement hors taxes et hors contingentements? Le long des territoires circulatoires leur proximité avec les pauvres des nations riches ou ceux des nations pauvres, les institue en colporteurs de l'économie du poor to poor désormais mondialisée. Objets d'une mobilisation internationale sur le mode ultra libéral, ni taxes ni frontières, intégrés à la haute densité sociale des territoires circulatoires, ils se vivent comme libres d'attaches

\footnotetext{
51 Des firmes prestigieuses produisent en masse, depuis les années 2000, des appareils photo (entre autres) d'entrée de gamme : vendu à 70 euros par la grande distribution européenne un Coolpix de Nikon est offert entre 30 et 35 euros " mano a mano " ou " tombé du camion " par nos transmigrants.

52 Baloutches afghans et iraniens notamment (voir Tarrius, 2012).
} 
aux nations qu'ils traversent. Paradoxalement les plus pauvres des circulants internationaux rejoignent et illustrent le projet mondial des firmes transnationales les plus riches, celui des libres circulations assorties de la fin des nations. Une culture populaire mondiale, le peer to peer, ou " entre experts " s'affirme conjointement : par exemple les jeunes des quartiers enclavés, comme ceux de tout autre habitat, se surinforment par l'internet et diverses revues sur les plus récentes productions d'entrée de gamme des majors du Sud-Est asiatique et deviennent les agents commerciaux du poor to poor.

Les majors de l'industrie du médicament semblent prendre la même voie, confiant leur déploiement à l'internet et aux logistiques usuelles, mais visant, eux aussi, le marché mondial des populations de pauvres qui échappent de plus en plus aux dispositifs de santé des États. Crises économiques nationales obligent. Là encore poor to poor et peer to peer se déploient rapidement.

La croisée des chemins des transmigrants et des migrants historiques illustre à l'échelle locale, les capacités de résilience populaire adossées aux stratégies planétaires des grandes firmes et suggère de nouveaux profils d'étrangers.

Les processus que nous décrivons, expressions de mobilités migratoires organisées selon le rapport transmigrations/mondialisation s'affirment et se déploient depuis les années 1980 en parallèle aux classiques et massives mobilités immigrations/nations. La modestie de leurs manifestations et l'invisibilité statistique de leurs présences ne doivent pas minorer leur importance dans l'évolution contemporaine de la figure de l'étranger. Georg Simmel, en premier lieu, puis toutes les approches compréhensives que nous signalons, ont démontré la richesse prédictive ou symptomatique de telles manifestations. Fin de la parenthèse des contextes nationaux industriels ?

\section{Références bibliographiques}

Barel Yves (Éd.) (1985) L'autonomie sociale aujourd'hui, Grenoble, PUG, CEPSCREA, $730 \mathrm{p}$.

Barel Yves (1979) Le paradoxe et le système. Essai sur le fantastique social, Grenoble, PUG, 275 p.

Battegay Alain (2003) Les recompositions d'une centralité commerçante immigrée : la Place du Pont à Lyon, Revue Européenne des Migrations Internationales, 19 (2), pp. 9-22.

Braudel Fernand (1979) [1949] La Méditerranée et le monde méditerranéen à l'époque de Philippe II, Paris, Armand Colin, 1160 p.

Carreno Jean-André, Hayot Alain et Lesme Francis (1974) Le quartier de la Porte d'Aix à Marseille. Essai d'ethnologie d'un centre urbain, Aix-en Provence, CERESM, $142 \mathrm{p}$.

Diminescu Dana (2005) Le migrant connecté. Pour un manifeste épistémologique, Migrations et Sociétés, 102, pp. 275-293.

Fontaine Laurence (2008) L'économie morale. Pauvreté, crédit et confiance dans I'Europe préindustrielle, Paris, Gallimard, $437 \mathrm{p}$. 
Foucault Michel (1967) Hétérotopies, hétérochronies, polycopié non publié, 12 p.

Goffman Irwin (1983) The interactive order, American Review of Sociology, 48 (18), pp. 1-17.

Gourdon Jean-Loup, Perrin Évelyne et Tarrius Alain (1995) Ville, espace, valeurs, Paris, L'Harmattan, 582 p.

Grossin William (1974) Les temps de la vie quotidienne, Paris-La Haye, Mouton, $416 \mathrm{p}$.

Gurvitch Georges (1963) La multiplication des temps sociaux, Paris, PUF, 365 p.

Halbwachs Maurice (1971) [1941] La topographie légendaire des Évangiles en Terre Sainte : étude de mémoire collective, Paris, PUF, $171 \mathrm{p}$.

Hannerz Ulf (1983) Explorer la ville, Paris, Les Éditions de Minuit, 432 p.

Heidegger Martin (1958) Essais et conférences, Paris, Gallimard, 378 p.

Husserl Edmond (1976) [1921] La crise des sciences européennes et la philosophie transcendantale, traduction de Gérard Granel et Jacques Derrida, Paris, Gallimard, $589 \mathrm{p}$.

Husserl Edmond (1931) Méditations cartésiennes, traduction d'Emmanuel Levinas et Gabrielle Peiffer, Paris, Armand Colin, 136 p.

Marcuse Herbert (1963) [1955] Eros et civilisation. Contribution à Freud, Paris, Les Éditions de Minuit, 244 p.

Mercure Daniel (1979) L'étude des temporalités sociales, Cahiers internationaux de sociologie, 67, pp. 263-276.

Merleau-Ponty Maurice (1976) Phénoménologie de la perception, Paris, Gallimard, $560 \mathrm{p}$.

Park Robert Ezra (1955) Society: Collective Behavior, News and Opinions, Sociology, and Modern Society, Glencoe III., The Free Press, 358 p.

Schütz Alfred (1988) Le chercheur et le quotidien, Paris, Méridiens, 286 p.

Tarrius Alain (2013) La mer Noire, moral area matrice de nouvelles migrations internationales en contexte de globalisation, Espaces Temps.net, [en ligne]. URL : http://www.espacestemps.net/articles/les-carrefours-migratoires-mondiaux/

Tarrius Alain (2012) 2001-2010. L'invention des routes commerciales des transmigrants baloutches de Kandahar à Malaga, Diasporas, histoire et sociétés, 20, pp. 131-148.

Tarrius Alain (2007) La remontée des Sud, Afghans et Marocains en Europe méridionale, LaTour-d'Aigues, l'Aube, 202 p.

Tarrius Alain (1995) Arabes de France dans l'économie souterraine mondiale, La Tour-d'Aigues, l'Aube, 219 p.

Tarrius Alain (1993) Territoires circulatoires et espaces urbains, Annales de la Recherche Urbaine, 52, pp. 51-60.

Tarrius Alain (1989) Anthropologie du mouvement, Caen, Paradigme, 185 p. 
Tarrius Alain (1987) L'entrée dans la ville, migrations maghrébines et recomposition des tissus urbains à Marseille et à Tunis, Revue Européenne des Migrations Internationales, 3 (1-2), pp. 131-147.

Tarrius Alain et Bernet Olivier (2014) Mondialisation criminelle. Frontière franco espagnole, de La Junquera à Perpignan [téléchargeable gratuitement sur demande à altarrius@gmail.com], 90 p.

Tarrius Alain, Missaoui Lamia et Qacha Fatima (2013) Transmigrants et nouveaux étrangers, Toulouse, PUM, $200 \mathrm{p}$.

Thomas William et Znaniecki Florian (1998) Le paysan Polonais en Europe et en Amérique. Récit de vie d'un migrant, préface de Pierre Tripier, Paris, Nathan, $446 \mathrm{p}$.

Winkin Yves (1989) Goffman, les moments et leurs hommes, Paris, Le Seuil, $256 \mathrm{p}$. 


\section{Alain Tarrius}

\section{Quand les territoires circulatoires des transmigrants traversent des quartiers enclavés de villes moyennes françaises}

Deux notions, le paradigme méthodologique de la mobilité et les territoires circulatoires, que l'auteur a proposées dans les années 1990, lui ont permis de décrire et d'analyser les transmigrations de populations pauvres originaires du Maghreb, des Proche et Moyen-Orient, du Caucase et des Balkans, vers l'Europe méridionale. Cet article restitue les sources sociologiques et anthropologiques qui ont présidé à leur construction et les faits contemporains de transmigrations qu'elles éclairent. Pour terminer, il relate l'apparition, dans des quartiers enclavés de villes moyennes françaises, au carrefour des territoires circulatoires de transmigrant(e)s du " poor to poor " mondial et de l'accueil résidentiel fourni par les descendants des immigrants des années 1970, de dispositifs sociaux originaux, résiliences populaires en temps de crise. Les accueils réciproques entre colporteurs internationaux et jeunes des quartiers enclavés suggèrent un renouvellement des thèmes de recherches sur les migrations et une redéfinition de la notion d'étranger.

\section{When the "territoires circulatoires" of Transmigrants Come across Enclosed Districts on French Middle Towns}

Two notions, the methological paradigm of mobility and "territoires circulatoires", which the author created in the 1990's allowed him to describe and analyze the transmigrations of poor populations born in the Maghreb, in the Near and Middle East, in the Caucasus and in the Balkans, towards Southern Europe. The present paper retraces the sociological and anthropological sources which presided over their construction and the facts contemporary with the transmigrations which they highlight. At the end, the paper retraces the arrival, in enclosed districts of French middle towns, at the crossroads of "territoires circulatoires", of poor to poor worldwide phenomena and of the residential reception given by the descendants of migrants arrived in the 1970's, of original social means, the ordinary people resilience born from times of crisis. The mutual reception between international and young people from enclosed districts suggest to renew the research themes on migrations and to revisit the notion of foreigner.

\section{Cuando los «territoires circulatoires» de los transmigrantes atraviesan barrios aislados de ciudades francesas de mediana talla}

Dos nociones, el paradigma metodológico de la movilidad y los «territoires circulatoires", propuestos por el autor del artículo en los años noventa, le permiten ahora describir y analizar las transmigraciones de poblaciones pobres originarias del Magreb, de Oriente Medio y Próximo, del Cáucaso y de los Balcanes, hacia la Europa meridional. Este artículo restituye las fuentes sociológicas y antropológicas que llevaron a su construcción y las hace contemporáneas de las transmigraciones sobre las que estas arrojan luz. Para terminar relata la aparición, en los barrios aislados de las ciudades francesas de mediana talla, en la encrucijada de "territoires circulatoires" de transmigrantes del "poor to poor" mundial y de la acogida residencial dada por los descendientes de inmigrantes de los años setenta, de dispositivos sociales originales, resiliencias populares en tiempo de crisis. Estas acogidas recíprocas de los temas de investigación sobre las migraciones y una redefinición de la noción de extranjero. 